\title{
Names of Bacteria Validly Published in 1980 and 1981
}

The names in the following list were validly published in 1980 and 1981, either by publication in an original paper in IJSB or, if published elsewhere, by announcement in IJSB. Each bacterial name is followed by the name(s) of the author(s) responsible for the name and then by the citation to the publication of the name in IJSB. One can easily discern the bacterial names that have nomenclatural standing at any given time by consulting the Approved Lists of Bacterial Names (Int. J. Syst. Bacteriol. 30:225-420, 1980), the latest cumulative list (January issue of each year), and the issues of IJSB published in the same year as the latest cumulative list.

The Editor

Acetivibrio Patel et al. 1980 (30:179)

A. cellulolyticus Patel et al. 1980 (30:179)

A. ethanolgignens Robinson and Ritchie 1981 (31:335)

Acholeplasma morum Rose et al. 1980 (30:653)

Achromobacter Yabuuchi et al. 1981 (31:478)

A. xylosoxidans Yabuuchi et al. 1981 (31:477)

Acidiphilium Harrison 1981 (31:331)

A. cryptum Harrison 1981 (31:331)

Actinomadura ferruginea Meyer 1981 (31:215)

A. libanotica Meyer 1981 (31:215)

A. macra Huang 1980 (30:565)

A. spiralis Meyer 1981 (31:215)

Aeromonas sobria Popoff and Véron 1981 (31:215)

Agitococcus Franzmann and Skerman 1981 (31:182)

A. lubricus Franzmann and Skerman 1981 (31:182)

Alcaligenes faecalis subsp. homari Austin et al. 1981 (31:72)

Alteromonas hanedai Jensen et al. 1981 (31:382)

A. putrefaciens Lee et al. 1981 (31:215)

Aquaspirillum magnetotacticum Maratea and Blakemore 1981 (31:454)

Azomonotrichon Thompson and Skerman 1981 (31:215)

A. macrocytogenes Thompson and Skerman 1981 (31:215)

Azorhizophilus Thompson and Skerman 1981 (31:215)

A. paspali Thompson and Skerman 1981 (31:215)

Azotobacter armeniacus Thompson and Skerman 1981 (31:215)

A. nigricans subsp. achromogenes Thompson and Skerman 1981 (31:215)

Bacillus schlegelii Schenk and Aragno 1981 (31:215)

Bacteroides gingivalis Coykendall et al. 1980 (30:563)

B. gracilis Tanner et al. 1981 (31:442)

B. macacae (Slots and Genco) Coykendall et al. 1980 (30:563)

B. melaninogenicus subsp. macacae Slots and Genco 1980 (30:84)

Beneckea gazogenes Harwood et al. 1980 (30:655)

B. nereida Harwood et al. 1980 (30:655)

Beijerinckia indica subsp. lacticogenes Thompson and Skerman 1981 (31:215)

$B$. derxia subsp. venezuelae Thompson and Skerman 1981 (31:215)

Brevibacterium iodinum Collins et al. 1981 (31:216)
Campylobacter concisus Tanner et al. 1981 (31:442)

C. sputorum subsp. mucosalis Lawson et al. 1981 (31:390)

Cedecea Grimont et al. 1981 (31:317)

C. davisae Grimont et al. 1981 (31:317)

C. lapagei Grimont et al. 1981 (31:317)

Cellulomonas cartae Stackebrandt and Kandler 1980 (30:186)

Chitinophaga Sangkhobol and Skerman 1981 (31:288)

C. pinensis Sangkhobol and Skerman 1981 (31:289)

Chromatium purpuratum Imhoff and Trüper 1980 (30:601)

Chromobacterium fluviatile Moss et al. 1981 (31:216)

Clostridium aceticum Gottschalk and Braun 1981 (31:476)

C. puniceum Lund et al. 1981 (31:216)

C. purinolyticum Dürre et al. 1981 (31:192)

Corynebacterium liquefaciens (Okabayashi and Masuo) Lanéelle et al. 1981 (30:544)

C. vitarumen (Bechdel et al.) Lanéele et al. 1980 (30:542)

Cytophaga heparina (Payza and Korn) Christensen $1980(30: 475)$

Deinococcaceae Brooks and Murray 1981 (31:356)

Deinococcus Brooks and Murray 1981 (31:354)

D. erythromyxa Brooks and Murray 1981 (31:358)

D. proteolyticus Brooks and Murray 1981 (31:357)

D. radiodurans Brooks and Murray 1981 (31:354)

D. radiophilus Brooks and Murray 1981 (31:357)

D. radiopugnans Brooks and Murray 1981 (31:358)

Desulfobacter Widdel 1981 (31:382)

D. postgatei Widdel 1981 (31:382)

Desulfobulbus Widdel 1981 (31:382)

D. propionicus Widdel 1981 (31:382)

Desulfococcus Widdel 1981 (31:382)

D. multivorans Widdel 1981 (31:382)

Desulfonema Widdel 1981 (31:382)

D. limicola Widdel 1981 (31:382)

D. magnum Widdel 1981 (31:382)

Desulfosarcina Widdel 1981 (31:382)

D. variabilis Widdel 1981 (31:382)

Desulfovibrio baarsii Widdel 1981 (31:382)

D. sapovorans Widdel 1981 (31:382)

Edwardsiella hoshinae Grimont et al. 1981 (31:216)

E. ictaluri Hawke et al. 1981 (31:400) 
Enterobacter amnigenus Izard et al. 1981 (31:37)

E. gergoviae Brenner et al. 1980 (30:1)

E. intermedium Izard et al. 1980 (30:601)

E. sakazakii Farmer et al. 1980 (30:569)

Eubacterium brachy Holdeman et al. 1980 (30:164)

E. nodatum Holdeman et al. 1980 (30:164)

E. timidum Holdeman et al. 1980 (30:164)

Flavobacterium multivorum Holmes et al. 1981 (31:22)

Flexibacter canadensis Christensen 1980 (30:431)

Fluoribacter Garrity et al. 1980 (30:612)

F. bozemanae Garrity et al. 1980 (30:612)

F. dumoffii (Brenner et al.) Brown et al. 1981 (31:114)

F. gormanii (Morris et al.) Brown et al. 1981 (31:114)

Frateuria Swings et al. 1980 (30:555)

F. aurantia Swings et al. 1980 (30:555)

Fusobacterium polysaccharolyticum van Gylswyk 1981 (31:382)

Gardnerella Greenwood and Pickett 1980 (30:176)

G. vaginalis (Gardner and Dukes) Greenwood and Pickett 1980 (30:176)

Halomonas Vreeland et al. $1980(30: 494)$

$H$. elongata Vreeland et al. 1980 (30:495)

Holospora Gromov and Ossipov 1981 (31:351)

H. obtusa Gromov and Ossipov 1981 (31:351)

$H$. undulata Gromov and Ossipov 1981 (31:351)

Klebsiella terrigena Izard et al. 1981 (31:125)

Kluyvera Farmer et al. 1981 (31:382)

$K$. ascorbata Farmer et al. 1981 (31:382)

K. cryocrescens Farmer et al. 1981 (31:382)

Lactobacillus amylophilus Nakamura and Crowell 1981 (31:216)

L. amylovorus Nakamura 1981 (31:61)

L. bavaricus Stetter and Stetter 1980 (30:601)

L. gasseri Lauer and Kandler 1980 (30:601)

Legionella bozemanii Brenner et al. 1980 (30:676)

L. dumoffii Brenner et al. 1980 (30:676)

L. gormanii Morris et al. 1980 (30:676)

L. micdadei Hebert et al. 1980 (30:676)

L. pittsburghensis Pasculle et al. 1980 (30:676)

Methanobacteriales Balch and Wolfe 1981 (31:216)

Methanobacterium bryantii Balch and Wolfe 1981 (31:216)

Methanobrevibacter Balch and Wolfe 1981 (31:216)

$M$. arboriphilicus (Zeikus and Henning) Balch and Wolfe 1981 (31:216)

$M$. ruminantium (Smith and Hungate) Balch and

Wolfe 1981 (31:216)

M. smithii Balch and Wolfe 1981 (31:216)

Methanococcaceae Balch and Wolfe 1981 (31:216)

Methanococcales Balch and Wolfe 1981 (31:216)

Methanococcus voltae Balch and Wolfe 1981 (31:216)

Methanogenium Romesser et al. 1981 (31:216)

M. cariaci Romesser et al. 1981 (31:216)

M. marisnigri Romesser et al. 1981 (31:216)

Methanomicrobiaceae Balch and Wolf 1981 (31:216)

Methanomicrobiales Balch and Wolf 1981 (31:216)

Methanomicrobium Balch and Wolf 1981 (31:216)

M. mobile (Paynter and Hungate) Balch and Wolf 1981

(31:216)

Methanosarcinaceae Balch and Wolf 1981 (31:216)
Methylococcus bovis Romanovskaya et al. 1981 (31:382)

M. chroococcus Romanovskaya et al. 1981 (31:382)

M. luteus Romanovskaya et al. 1981 (31:382)

M. mobilis Hazeu et al. 1980 (30:676)

M. vinelandii Romanovskaya et al. 1981 (31:382)

M. whittenburyi Romanovskaya et al. 1981 (31:382)

Morococcus Long et al. 1981 (31:299)

$M$. cerebrosus Long et al. (31:299)

Mycobacterium agri Tsukamura 1981 (31:256)

M. aichiense Tsukamura et al. 1981 (31:274)

$M$. chubuense Tsukamura et al. 1981 (31:274)

M. obuense Tsukamura et al. 1981 (31:274)

M. rhodesiae Tsukamura et al. 1981 (31:274)

$M$. sphagni Kazda 1980 (30:81)

M. tokaiense Tsukamura et al. 1981 (31:274)

Mycoplasma californicum Jasper et al. 1981 (31:344)

M. fastidiosum Lemcke and Poland 1980 (30:161)

Pasteurellaceae Pohl 1981 (31:382)

Photobacterium logei Harwood et al. 1980 (30:655)

Planctomyces maris Bauld and Staley 1980 (30:595)

Prosthecobacter Staley et al. 1980 (30:595)

P. fusiformis Staley et al. 1980 (30:595)

Pseudomonas carboxydohydrogena Meyer et al. 1980 (30:194)

P. cepacia Palleroni and Holmes 1981 (31:479)

$P$. corrugata Roberts and Scarlett 1981 (31:216)

$P$. maltophilia Hugh 1981 (31:195)

P. meliae Ogimi 1981 (31:382)

Rahnella Izard et al. 1981 (31:382)

$R$. aquatilis Izard et al. 1981 (31:382)

Renibacterium Sanders and Fryer 1980 (30:501)

R. salmoninarum Sanders and Fryer 1980 (30:501)

Rhodopseudomonas blastica Eckersley and Dow 1981 (31:216)

Serratia ficaria Grimont et al. 1981 (31:216)

Spirochaeta aurantia Canale-Parola 1980 (30:594)

S. littoralis Canale-Parola 1980 (30:594)

S. zuelzerae Canale-Parola 1980 (30:595)

Spiroplasma floricola Davis et al. 1981 (31:462)

Streptomyces armeniacus (Kalakoutskii and Kusnetsov) Wellington and Williams 1981 (31:80)

S. echinoruber Palleroni et al. 1981 (31:382)

S. longwoodensis Prosser and Palleroni 1981 (31:382)

S. sannanensis Iwasaki et al. 1981 (31:283)

Sulfolobus brierleyi Zillig et al. 1980 (30:676)

$S$. solfataricus Zillig et al. 1980 (30:676)

Tatlockia Garrity et al. 1980 (30:612)

T. micdadei (Hebert et al.) Garrity et al. 1980 (30:612)

Thermoactinomyces intermedius Kurup et al. 1981 (31:216)

Thermothrix Caldwell et al. 1981 (31:217)

T. thiopara Caldwell et al. 1981 (31:217)

Treponema bryantii Stanton and Canale-Parola 1980 (30:676)

T. succinifaciens Cwyk and Canale-Parola 1981 (31:383)

Vampirovibrio Gromov and Mamkayeva 1980 (30:676)

V. chlorellavorus Gromov and Mamkayeva 1980 (30:676) 
Vibrio campbellii (Baumann et al.) Baumann et al. 1981 (31:217)

V. fluvialis Lee et al. 1981 (31:217)

V. gazogenes (Harwood et al.) Baumann et al. 1981 (31:217)

$V$. harveyi (Reichelt and Baumann) Baumann et al. 1981 (31:217)

V. logei (Harwood et al.) Baumann et al. 1981 (31:217)

$V$. natriegens (Baumann et al.) Baumann et al. 1981 (31:217)

V. nereis (Harwood et al.) Baumann et al. 1981 (31:217)

$V$. nigripulchritudo (Baumann et al.) Baumann et al. 1981 (31:217)
V. pelagius (Baumann et al.) Baumann et al. 1981 (31:217)

V. splendidus (Reichelt et al.) Baumann et al. 1981 (31:217)

V. vulnificus Farmer 1980 (30:656)

Wolinella Tanner et al. 1981 (31:439)

W. recta Tanner et al. 1981 (31:441)

W. succinogenes (Wolin et al.) Tanner et al. 1981 (31:439)

Yersinia frederiksenii Ursing et al. 1981 (31:217)

$Y$. intermedia Brenner et al. 1981 (31:217)

Y. kristensenii Bercovier et al. 1981 (31:217)

Y. pseudotuberculosis subsp. pestis (van Loghem) Bercovier et al. 1981 (31:383)

List of Specific and Subspecific Epithets

aceticum (Clostridium)

achromogenes (Azotobacter nigricans subsp.)

agri (Mycobacterium)

aichiense (Mycobacterium)

amnigenus (Enterobacter)

amylophilus (Lactobacillus)

amylovorus (Lactobacillus)

aquatilis (Rahnella)

arboriphilicus (Methanobrevibacter)

armeniacus (Azotobacter, Streptomyces)

ascorbata (Kluyvera)

aurantia (Frateuria, Spirochaeta)

baarsii (Desulfovibrio)

bavaricus (Lactobacillus)

blastica (Rhodopseudomonas)

bovis (Methylococcus)

bozemanae (Fluoribacter)

bozemanii (Legionella)

brierleyi (Sulfolobus)

bryantii (Methanobacterium, Treponema)

californicum (Mycoplasma)

campbellii (Vibrio)

canadensis (Flexibacter)

carboxydohydrogena (Pseudomonas)

cariaci (Methanogenium)

cartae (Cellulomonas)

cellulolyticus (Acetivibrio)

cepacia (Pseudomonas)

cerebrosus (Morococcus)

chlorellavorus (Vampirivibrio)

chroococcus (Methylococcus)

chubuense (Mycobacterium)

concisus (Campylobacter)

corrugata (Pseudomonas)

cryocrescens (Kluyvera)

cryptum (Acidiphilium)

davisae (Cedecea)

dumoffi (Fluoribacter, Legionella)

echinoruber (Streptomyces)

elongata (Halomonas)

erythromyxa (Deinococcus)

ethanolgignens (Acetivibrio)

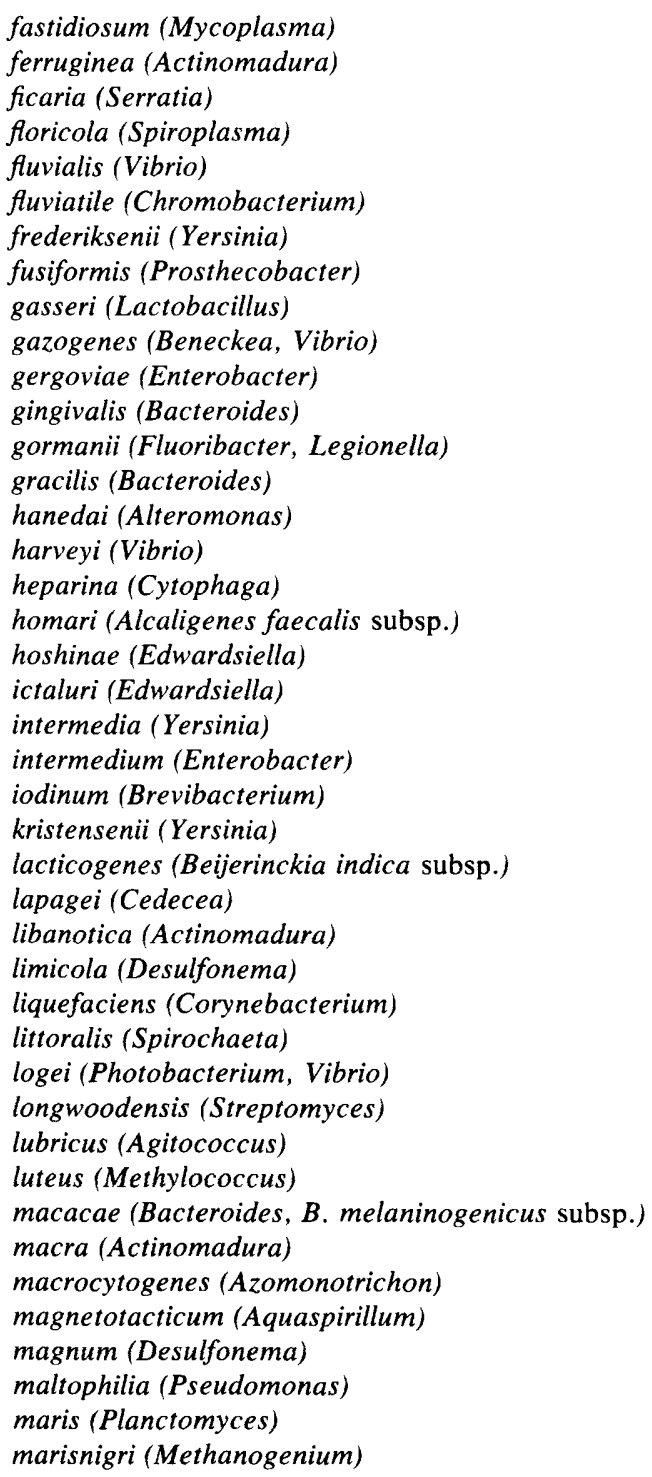

fastidiosum (Mycoplasma)

fluvialis (Vibrio)

fluviatile (Chromobacterium)

frederiksenii (Yersinia)

fusiformis (Prosthecobacter)

gasseri (Lactobacillus)

gergoviae (Enterobacter)

gingivalis (Bacteroides)

gormanii (Fluoribacter, Legionella)

gracilis (Bacteroides)

harveyi (Vibrio)

heparina (Cytophaga)

homari (Alcaligenes faecalis subsp.)

nae (Edwardsiella)

intermedium (Enterobacter)

iodinum (Brevibacterium)

kristensenii (Yersinia)

lapagei (Cedecea)

libanotica (Actinomadura)

limicola (Desulfonema)

liquefaciens (Corynebacterium)

littoralis (Spirochaeta)

lubricus (Agitococcus)

luteus (Methylococcus)

macacae (Bacteroides, B. melaninogenicus subsp.)

marisnigri (Methanogenium) 
meliae (Pseudomonas)

micdadei (Legionella, Tatlockia)

mobile (Methanomicrobium)

mobilis (Methylococcus)

morum (Acholeplasma)

mucosalis (Campylobacter sputorum subsp.)

multivorans (Desulfococcus)

multivorum (Flavobacterium)

natriegens (Vibrio)

nereida (Beneckea)

nereis (Vibrio)

nigripulchritudo (Vibrio)

nodatum (Eubacterium)

obtusa (Holospora)

obuense (Mycobacterium)

paspali (Azorhizophilus)

pelagius (Vibrio)

pestis (Yersinia pseudotuberculosis subsp.)

pinensis (Chitinophaga)

pittsburghensis (Legionella)

polysaccharolyticum (Fusobacterium)

postgatei (Desulfobacter)

propionicus (Desulfobulbus)

proteolyticus (Deinococcus)

puniceum (Clostridium)

purinolyticum (Clostridium)

purpuratum (Chromatium)

putrefaciens (Alteromonas)

radiodurans (Deinococcus)

radiophilus (Deinococcus)

radiopugnans (Mycobacterium) recta (Wolinella)

rhodesiae (Mycobacterium)

ruminantium (Methanobrevibacter)

sakazakii (Enterobacter)

salmoninarum (Renibacterium)

sannanensis (Streptomyces)

sapovorans (Desulfovibrio)

schlegelii (Bacillus)

smithii (Methanobrevibacter)

sobria (Aeromonas)

solfataricus (Sulfolobus)

sphagni (Mycobacterium)

spiralis (Actinomadura)

splendidus (Vibrio)

succinifaciens (Treponema)

succinogenes (Wolinella)

terrigena (Klebsiella)

thiopara (Thermothrix)

timidum (Eubacterium)

tokaiense (Mycobacterium)

undulata (Holospora)

vaginalis (Gardnerella)

variabilis (Desulfosarcina)

venezuelae (Beijerinckia derxia subsp.)

vinelandii (Methylococcus)

vitarumen (Corynebacterium)

voltae (Methanococcus)

vulnificus (Vibrio)

whittenburyi (Methylococcus)

xylosoxidans (Achromobacter)

zuelzerae (Spirochaeta) 